\title{
Review
}

\section{Eco-Efficient Mobility in Smart City Scenarios}

\author{
Ramon Sanchez-Iborra ${ }^{1}\left(\mathbb{D}\right.$, Luis Bernal-Escobedo ${ }^{1}(\mathbb{B})$ and José Santa ${ }^{2, *}$ (i) \\ 1 Department of Information and Communication Engineering, University of Murcia, 30100 Murcia, Spain; \\ ramonsanchez@um.es (R.S.-I.); luis.bernal@um.es (L.B.-E.) \\ 2 Department of Electronics, Computer Technology and Projects, Technical University of Cartagena, \\ 30202 Cartagena, Spain \\ * Correspondence: jose.santa@upct.es
}

Received: 23 September 2020; Accepted: 9 October 2020; Published: 14 October 2020

\begin{abstract}
Cooperative-Intelligent Transportation Systems (C-ITS) have brought a technological revolution, especially for ground vehicles, in terms of road safety, traffic efficiency, as well as in the experience of drivers and passengers. So far, these advances have been focused on traditional transportation means, leaving aside the new generation of personal vehicles that are nowadays flooding our streets. Together with bicycles and motorcycles, personal mobility devices such as segways or electric scooters are firm sustainable alternatives that represent the future to achieve eco-friendly personal mobility in urban settings. In a near future, smart cities will become hyper-connected spaces where these vehicles should be integrated within the underlying C-ITS ecosystem. In this paper, we provide a wide overview of the opportunities and challenges related to this necessary integration as well as the communication solutions that are already in the market to provide these moving devices with low-cost and efficient connectivity. We also present an On-Board Unit (OBU) prototype with different communication options based on the Low Power Wide Area Network (LPWAN) paradigm and several sensors to gather environmental information to facilitate eco-efficiency services. As the attained results suggest, this module allows personal vehicles to be fully integrated in smart city environments, presenting the possibilities of LoRaWAN and Narrow Band-Internet of Things (NB-IoT) communication technologies to provide vehicle connectivity and enable mobile urban sensing.
\end{abstract}

Keywords: personal mobility; eco-efficient vehicles; smart city; internet of vehicles; C-ITS

\section{Introduction}

While transport emissions start decelerating worldwide, they are still responsible for $24 \%$ of $\mathrm{CO}_{2}$ emissions from fuel combustion [1]. There are a multitude of works in the literature dealing with this issue in maritime [2,3] and air transport [4]. However, it is the road segment, including heavy and light vehicles, the one that accumulates $3 / 4$ of transport emissions [1]. These values are extremely high under a global warming far from being solved. Electric vehicles appear as a potential solution to gradually alleviate this problem. Their sales have increased exponentially last years, involving not only cars, but also two-wheelers. As indicated in [5], about $20 \%$ of these vehicles are electric right now, with special mention given to electric micro-mobility, including bikes and scooters. This opens a new research niche in which to apply new information and communication technologies to improve traffic efficiency and assure safety, at the time that emissions are reduced in urban areas, above all.

During the last twenty years, we have witnessed prominent advances in the vehicular industry. A clear example is the field of autonomous driving that, by the use of state-of-the-art on-board sensing and electronic systems, has become a reality today. Autonomous navigation systems have been refined by the integration of connectivity solutions, which also provide vehicles with 
novel services supported by new-generation communication networks [6]. From the perspective of Cooperative-Intelligent Transportation Systems (C-ITS), vehicles are able to communicate among each other (Vehicle-to-Vehicle, V2V) and with the infrastructure (Vehicle-to-Infrastructure, V2I), as shown in Figure 1. This allows for widening the surroundings perception of each vehicle and has fueled the development of new productivity, safety, and entertainment applications, which are the pillars of the future C-ITS ecosystem [7]. However, it is a fact that these advances have been devoted to traditional vehicles, therefore ignoring the previously cited emergent personal mobility options that are transforming the landscape of urban scenarios. Many citizens are currently making use of personal vehicles such as bicycles, scooters, or electric motorcycles, due to their attractive eco-efficient features and the promotion of sustainable and healthy lifestyles.

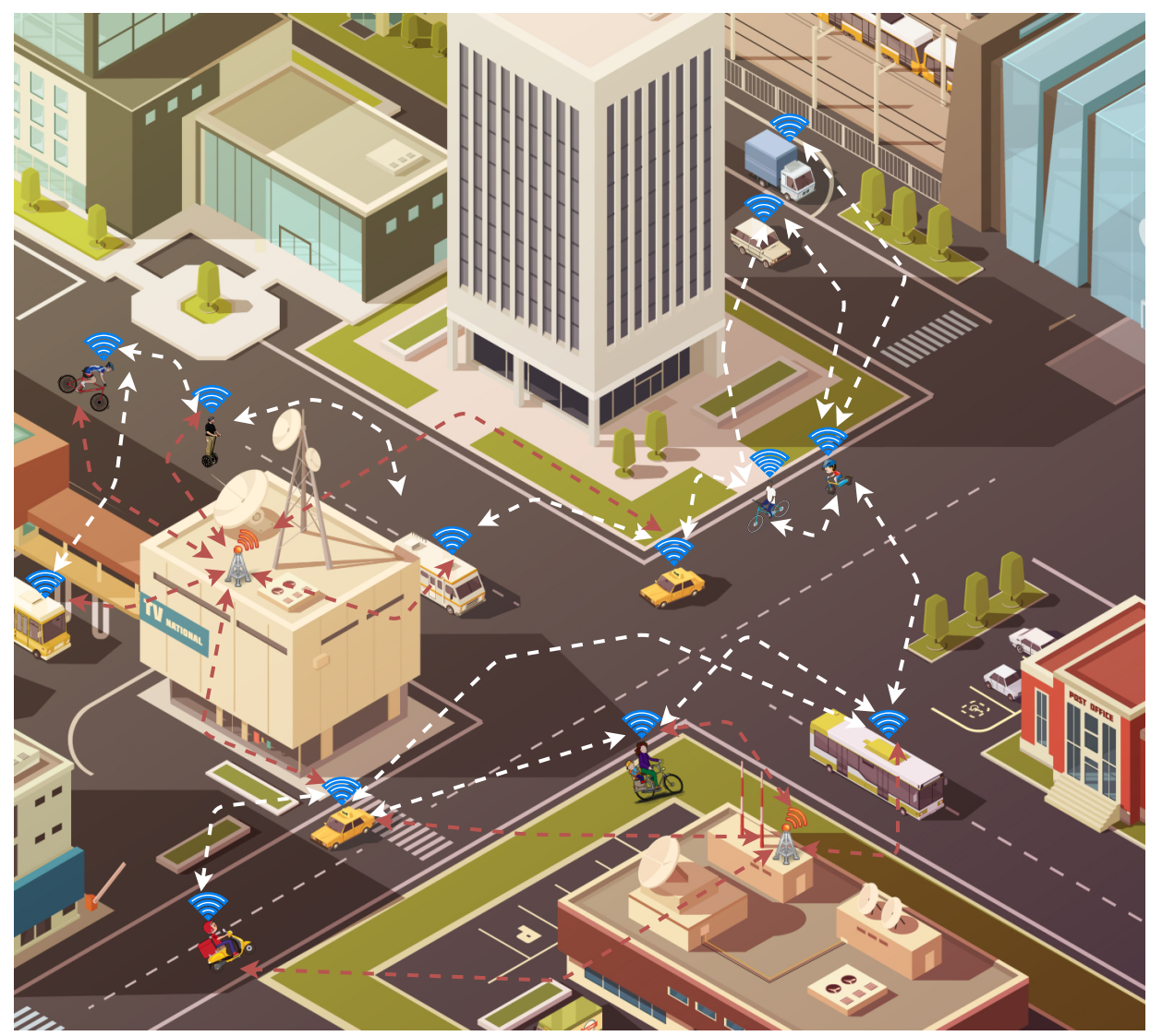

Figure 1. Urban fully-connected vehicular scenario. White/red arrows: V2V/V2I links.

In this particular vehicular segment, and focusing on new-age personal mobility devices, there are special issues to be tackled in both hardware and software planes. Firstly, On-Board Units (OBUs) should be designed considering stringent restrictions in terms of power consumption and hardware integration in a constrained vehicle body. Secondly, the software plane calls for adapted interfaces, safety-aware designs, and the integration of digital maps addressing the unique mobility characteristics of these vehicles. In general, new on-board solutions are needed to support a new range of applications in the frame of sustainable mobility. For those reasons, novel hardware and software platforms should be adapted for personal vehicles (usually two-wheleers) that will permit the integration of connected mopeds, bikes, segways, and equivalents into C-ITS and smart city scenarios. In this line, connectivity technologies coming from the Internet of Things (IoT), which have not been deeply investigated for vehicular scenarios yet, are suitable alternatives for this type of vehicles, given their aforementioned constraints. One clear example is the Low Power Wide Area Network (LPWAN) technology, which is 
being extensively adopted in smart city deployments, as it provides long range and energy efficient transmissions [8].

In this article, we provide a wide overview of the opportunities and challenges that the new wave of eco-efficient vehicles brings. We discuss about the specific needs of these elements as well as the potential services to be enabled for this market segment. The role of these new "moving things" in the game of urban sensing and big-data exploitation is also examined, as boosters of sustainable mobility and environment preservation. From this discussion, the great momentum of personal mobility is extracted, especially in urban scenarios, and its further development potential. However, a lack of dedicated OBU solutions for these particular family of vehicles in order to make them connected and intelligent is also detected. For that reason, we present an OBU prototype integrating a series of sensing devices and communication interfaces, which has been installed and tested on board an electric scooter. The results obtained in our validation tests reveal the efficacy of the design. In addition, we explore the performance of two prominent LPWAN technologies that have been embedded in our prototype, namely, LoRAWAN and Narrow Band-IoT (NB-IoT), showing their different characteristics and how each of them is more appropriate for certain types of services. Overall, the connectivity capabilities provided by our OBU prototype enables the integration of sustainable vehicles within the upcoming age of C-ITS and smart cities.

The rest of the paper is organized as follows. Section 2 reviews recent works related to the personal vehicle ecosystem. A comprehensive discussion regarding the opportunities and challenges as well as enabling communication technologies for personal vehicles are presented in Section 3. Section 4 describes the proposed OBU prototype and examines the validation and performance results. Finally, the paper is concluded in Section 5 summarizing the most important finding and drawing future research lines.

\section{Related Work}

As stated previously, although many works have explored the field of vehicular networks [9] and their related services [10], there is a lack of papers addressing the potential of the novel personal mobility ecosystem as a whole. However, some specific efforts have been devoted in the field of vehicular data gathering for enabling the cooperation among different-nature mobile devices.

Data gathering in Vehicular Sensor Networks (VSNs) has partially addressed the issue of saving communication costs, such as the work in [11], which tried to reduce data rate by considering a trade-off with the required freshness of sensor reads for a concrete application. In [12], 4G communication was combined with WiFi offloading and a crowd-sensing approach, using regular smart phones as a communication relay. An equivalent approach with a mobile phone was adopted in [13] for monitoring vehicle emissions, and [14], which employed Bluetooth to interconnect on-board sensors. Recent proposals such as [15] took advantage of the mesh created by vehicular (ad-hoc) networks to spread mobile agents in charge of gathering data from a target area.

Work in [16] proposed a holistic data gathering and processing platform for smart cities leveraging the potential of the IoT paradigm. The different connected objects included smart phones, infrastructure fixed stations as well as OBUs carried by public buses. Authors employed both 802.11 Outside the Context of a Basic Service Set (OCB), formerly known as 802.11p, and cellular communications, namely, 4G. A similar solution was presented in [17], where state-of-the-art visible light networks were also employed as a complementary data path.

Regarding the development of OBUs considering the constraints of personal vehicles, few contributions can be found in the literature. Authors of [18] proposed the use of ZigBee-based connectivity in bicycle OBUs. The aim of this solution was to report sensed data and create convoys of bikes in order to improve their safety. In [19], Bluetooth was employed with a similar purpose, evidencing the limited communication range of this type of technologies. The authors of [20] developed a motorbike OBU using WiFi OCB, a technology that was also investigated in [21]. In addition, the latter also made use of an enhanced IEEE 802.15.4 link for V2V transmissions. 
Considering human-machine interaction, work in [22] presented an advanced navigation assistance system for bicycles by means of a vibrating belt and cellular communications. In turn, the authors of [23] also explored the direct interaction of the vehicle with the driver by connecting a helmet to an OBU implemented by means of a smart phone with cellular connectivity.

Finally, it is worth mentioning some crowd-sensing solutions that have been specifically designed for bikes. An Arduino-based platform was adopted in [24] to retrieve and report pollution data employing cellular communications. The authors of [25] developed a similar sensing platform although they made use of a smart phone tethering to transmit the gathered data. A similar solution was presented in [26], in this case employing LoRa and WiFi as communication technologies.

It is clear that the contributions to the field of sustainable personal mobility are limited. However, these vehicles are more and more common in our cities. For that reason, in this paper, we provide a detailed discussion addressing the potential opportunities and challenges brought by this specific vehicular segment. Future services, communication technologies, and current gaps within this novel ecosystem are explored. In addition, a novel OBU architecture and prototype considering the stringent requirements of these moving devices are proposed and a series of validation tests are presented, showing the performance of the solution using LPWAN communication technologies.

\section{Personal Vehicles and C-ITS}

\subsection{Services for Personal Vehicles and Beyond}

It is forecast that almost 70\% of world's population will live in cities by 2050 [27]. This implies a real need for sustainable ways of transportation in order to make cities better places to live. In fact, electric personal vehicles are already replacing cars and motorbikes in urban scenarios, which has a positive impact on the urban mobility's carbon footprint and, consequently, on the city inhabitants' health. Switching from a car to eco-friendly alternatives is nowadays simple and satisfactory thanks, in part, to connectivity-and this is only the beginning. The inclusion of these vehicles into hyper-connected spaces, as shown in Figure 1, offers users a plethora of novel practical services that improve their eco-mobility experience and safety.

For obvious reasons, traffic safety has been the main focus of research related to vehicular connectivity during the last few years. Advances in this field could be even more important in the case of personal vehicles due to their vulnerability. Although many efforts are being done by city councils for separating bicycles and equivalents from regular traffic, they are still the weakest link in the chain. So far, due to the great latency of V2I communication, V2V alternatives have been employed to warn riders about dangerous proximity of another vehicle [28]. However, with the arrival of 5G, it is expected that the infrastructure could also help with improving road safety with a comprehensive vision of traffic and roads in real time.

Intelligent route planning is another relevant service that permits finding the shortest and safest routes towards a given destination or even suggesting intermodality alternatives taking into account user needs or preferences [29]. In this line, route planners may adopt different path choice strategies considering the dynamic conditions of unreliable transit networks [30]. In addition, by integrating these kinds of systems in a smartphone, the application may guide the user before and after the ride, hence helping her to find an available vehicle (bike, scooter, etc.) or a valid parking spot in the case of using a vehicle-sharing service. These services are great beneficiaries of online vehicle location and tracking systems aiming at developing effective fleet management systems as well as improving its economic feasibility [31]. This is of prominent importance for achieving a coherent fleet organization and distribution but also for providing customers with a service cloud-platform to enable reservations or provide real-time traffic analytics, among other useful services.

Digitalizing private vehicles also opens a range of valuable applications for owners, public authorities and citizens, in general. The first that comes to mind is security. Thousands of bikes and scooters are stolen every day in cities worldwide. Connectivity solutions can strengthen 
the classic mechanical lock, not only by enabling their real-time tracking as mentioned above, but also by using the OBU as a smart-lock managing vehicle's electronics. These e-lock capabilities can make the device unusable if manipulated, hence making the vehicle less attractive in the reselling market. In addition, continuous vehicle monitoring permits to be in contact with the retailer or technical service by anticipating device failures or receiving advice or alerts from them.

Generalizing information collection from these vehicles, a plethora of new services in the area of traffic efficiency, environmental preservation, and citizen well-being can be fed with data harvested in Internet of Vehicle (IoV) scenarios. Sensory OBUs can monitor air pollution, sun radiation, noise, or navigation data, to analyze moving patterns, detect unhealthy areas, and recommend green itineraries or balance roads, cycle lanes and walking areas to reach a trade-off among all citizens.

Finally, the synergy of sustainable personal mobility with social media platforms is also highly interesting as it permits creating a sense of community. Through these services, users can share ideas, recommendations, or tips aligned with eco-efficient lifestyles. Fitness-tracking social tools are also included in this rich ecosystem to inform users about their daily workout and performance, and to find others with similar sportive interests. In addition, following the infotainment perspective of IoV, these platforms can also provide multimedia applications with the aim of improving the riding experience with music, tourist guides, audiobooks, etc.

\subsection{Gap Analysis}

As seen, the range of novel services from which personal vehicles will benefit is extensive. Therefore, we are facing a new scenario in which the integration of this range of personal mobility devices within smart cities and C-ITS ecosystems becomes a real demand from the society. However, research on communications and telematics in personal vehicles is notably limited so far. Previous works usually put two-wheelers at the same level of pedestrians, with the requirement differences and clear consequences that this implies [32]. Some papers have proposed the use of the cellular infrastructure for exchanging data with servers that emit safety warnings in case of potential collision with vulnerable road users [33]. Given the latency introduced by this technology, other proposals have adopted WiFi to reduce the system response time. However, due to the high power consumption of these technologies, some works have suggested the use of Zigbee [18] or Bluetooth [19], although the short communication range of these technologies certainly limits its general application in widespread vehicular scenarios. Finally, other authors bet on vehicular-specific communications, using IEEE OCB. However, interference issues should be considered in highly-crowded scenarios such as the urban one, together with strident battery constraints of personal mobility devices [34].

In the light of the previous discussion, an important set of challenges to be addressed is observed to provide eco-efficient personal vehicles with appropriate communication capabilities:

- Lack of specific hardware/software designs for bicycles, scooters, and similar vehicles.

- Efforts are focused on cellular or IEEE 802.11 OCB technologies, but not on hybridizing different communication technologies, adopting a Multi Radio Access Technology (multi-RAT) approach.

- The interconnection of these vehicles with IPv6 networks has not been addressed, which is crucial for their full integration in the Future Internet.

- The use of standardized protocols is not generalized, which is of prominent importance given the long-term life-cycles of the target vehicles.

- To the best of the authors' knowledge, there are no works exploiting the use of LPWAN technologies for personal vehicle monitoring, tracking, or eventual notification delivery.

- There are few proposals fostering transport intermodality and sustainable mobility options.

- Considering personal mobility devices as part of the future crowd-sensing ecosystem implies new IoV capabilities and sensory OBU platforms not present at the moment.

In the following, we present a wide overview of the vehicular communication landscape, discussing how some specific solutions can cover the identified gaps, as shown in Figure 2. 


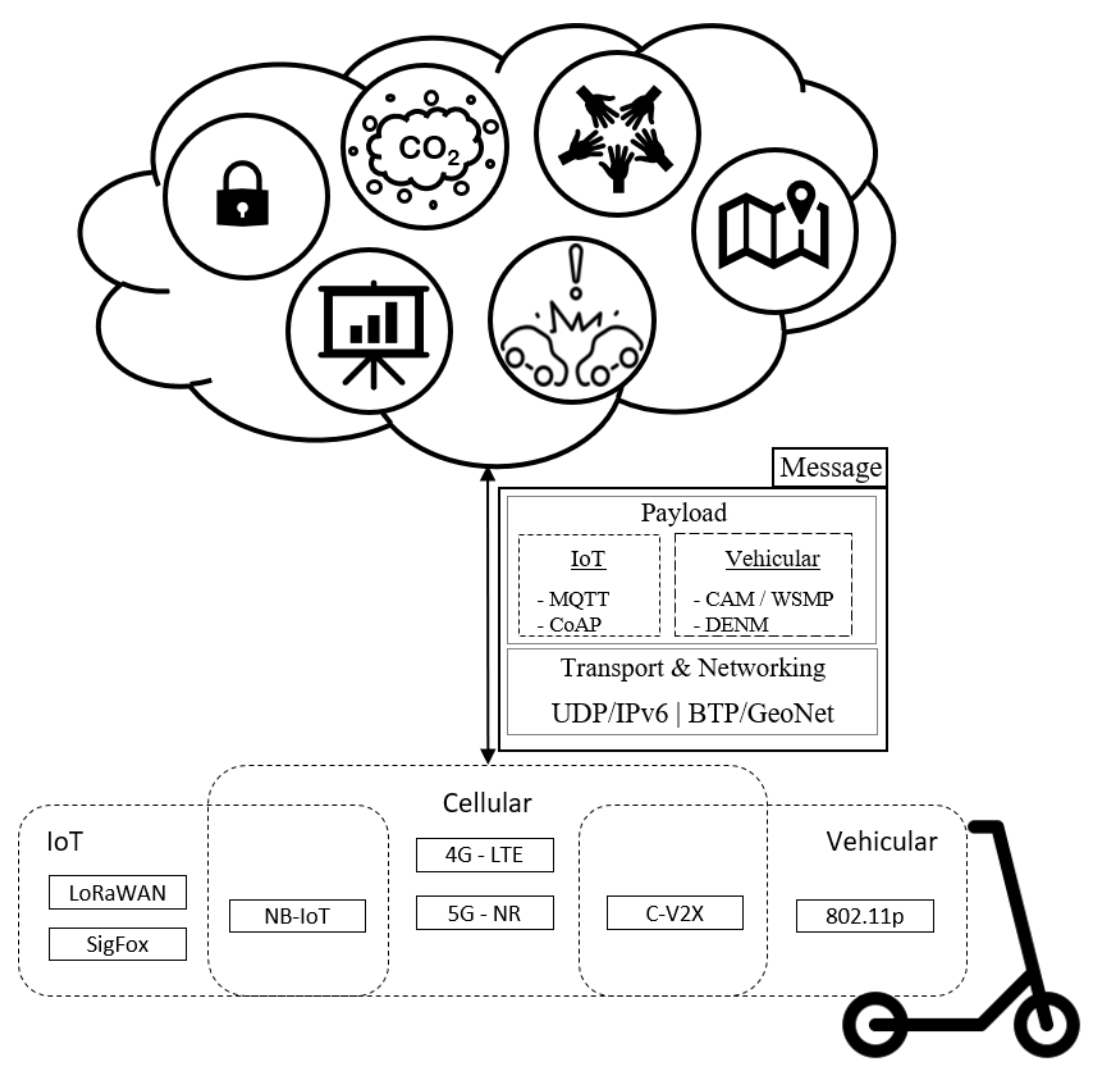

Figure 2. Cloud services for personal mobility devices, radio access technologies, and protocols.

\subsection{Communication Technologies}

Intelligent open spaces such as smart cities or smart university campuses aim at offering users a range of continuous services that should be seamlessly provided even considering end-user mobility. Without a doubt, the evolution of wireless communication technologies has enabled the development of the C-ITS ecosystem during the last decade. The first advances in digital cellular networks (2.5G and 3G) allowed the development of fleet management and monitoring applications for professional transport such as trucks, taxis, or coach buses. These services have been enriched with more advanced features, e.g., goods or driver tracking, with the arrival of 4G. Other services developed under this umbrella are real-time traffic information, automatic emergency call, or infotainment applications, among others. Therefore, it can be seen how cellular solutions have taken advantage for supporting delay-tolerant applications [35]. In addition, they make use of an already deployed architecture and the provided coverage in urban areas is usually enough for the offered services. Nevertheless, some concerns arise regarding the scalability of these networks when supporting thousands of nodes simultaneously connected to a single cell as well as the latency issues mentioned above. These problems disable current cellular solutions to support critical services such as crash avoidance. However, it is envisioned that the $5 \mathrm{G}$ technology will solve these issues with its guaranteed support to three types of services: enhanced Mobile Broadband (eMBB), Ultra Reliable Low Latency Communications (URLLC), and massive Machine Type Communications (mMTC) [36]. This promising technology is still in its infancy and the years to come will show whether functional deployments meet expectations.

Therefore, in the plane of traffic safety and dedicated transmissions, the technology with a greater level of maturity is IEEE OCB (formerly known as IEEE 802.11p), which is the main pillar of protocols such as the ones within the IEEE Wireless Access in Vehicular Environments (WAVE) and the general architecture defined by ISO/ETSI, as shown in Figure 2. Differently from the cellular alternatives, IEEE OCB also supports V2V communications, which permits notably reducing latency of communications with surrounding vehicles. In fact, the latest specification of 5G technology (release 15) includes dedicated communications for vehicular scenarios, considering integrated cellular vehicle to 
everything (C-V2X) connectivity. As aforementioned, although IEEE OCB has received great attention, specially from the Academia [37], its implantation has not been effective due to need for a fixed infrastructure, which has a notable cost given the great number of RSUs needed for covering a city [34]. In addition, this technology is energy-consuming for power-constrained devices, and it is prone to interferences and transmission collisions in highly crowded areas, which makes it unreliable for urgent information exchanges or even IoV monitoring scenarios in urban settings.

If this landscape is not complex enough, the new wave of IoT-related communication solutions has also come into play. The LPWAN paradigm promises long-range connectivity with limited energy consumption at the expense of offering limited data rates. Hence, as can be seen in Figure 2, the complementary use of LPWAN technologies arises as an interesting option for avoiding saturation of IEEE OCB and regular cellular channels due to not-urgent messages. Examples of LPWAN technologies are (i) NB-IoT, which is a solution integrated within the cellular ecosystem; (ii) LoRaWAN, which permits manageable private large-scale deployments; or (iii) Sigfox, which is a proprietary solution with an already deployed infrastructure in America and Europe [8].

Most of the services identified above for new-age personal vehicles may be supported by LPWAN-based technologies. They do not require either great bandwidth or low latency to provide a good Quality of Service (QoS). Tracking or monitoring applications usually make use of few small packets per minute, which is perfectly assumable by these connectivity solutions. The same occurs for security systems that just need a limited number of messages per transaction for locking or unlocking a device. In addition, some applications that do not require real-time data visualization may adopt data-aggregation strategies. For example, sensing applications that take environmental measurements as a public service, e.g., pollution, temperature, noise-level, etc., may aggregate collected data until the end of the ride and then send all the information to the cloud server by using few long transmissions.

As seen, LPWAN solutions are a firm alternative to provide personal vehicles with connectivity, hence enabling a plethora of novel services without harming other more urgent communication channels such as IEEE OCB or 5G, which should be devoted to critical applications.

\section{Proposed Solution}

\subsection{Architecture}

The conceptual design of the proposed OBU for sustainable personal vehicles is depicted in Figure 3. As observed, it is equipped with two different LPWAN-based technologies, namely, LoRaWAN and NB-IoT, which provide access to the Internet. Through these links, the device establishes bidirectional connections with servers in the cloud for reporting data and receiving information or commands. These servers can be accessed from personal mobile devices such as smart phones or tablets, to offer functions like those described previously, i.e., environmental monitoring, intelligent route guidance, security applications, etc.

There are two fundamental elements composing the core of the unit: the main board and the communication board. The former is an embedded single board computer that includes a regular CPU or microcontroller, and memory, integrated in a System on Chip (SoC) fashion. In this case, memory accounts for both RAM and flash memories. Both Inter-Integrated Circuit (I2C) and USB buses are considered in order to communicate this board with external modules, i.e., sensors and a communication concentrator. Some sensors could be also connected to direct digital or analog input/output lines. Whereas sensors are simple devices including (i) an inertial unit, (ii) a radiation detector, (iii) a noise meter, (iv) an environmental sensor (temperature, humidity and pressure), and (v) a pollution monitor, the communication concentrator is the second principal element of the OBU. It integrates a USB-to-serial adapter to enable communications by including chips such as GPS and the respective transceivers for the two selected LPWAN technologies: LoRaWAN and NB-IoT. These modules incorporate their corresponding external antennas to obtain better signal levels. 
The OBU is completed with an external display to show status messages and a battery unit to power the whole set.

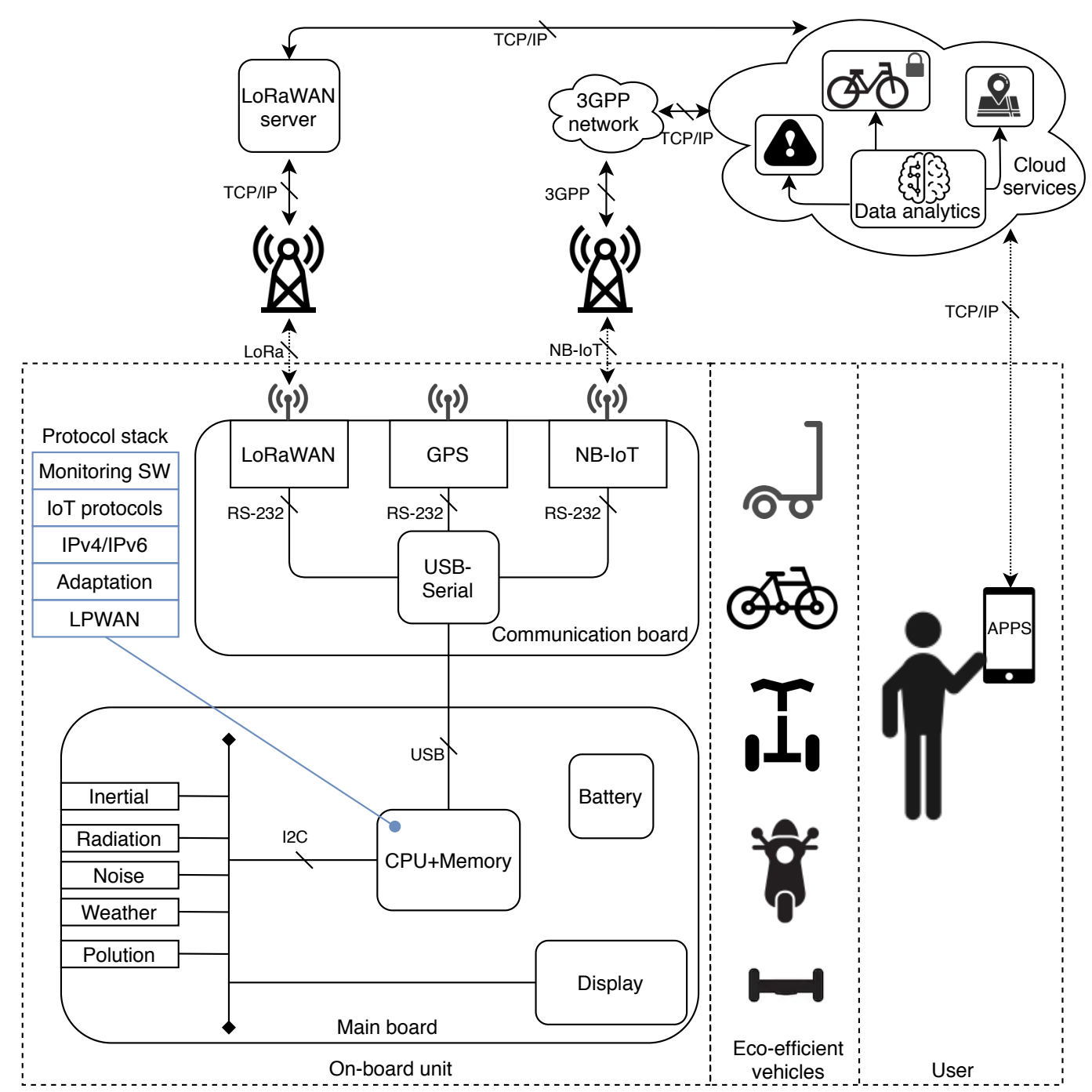

Figure 3. Architecture of the connectivity solution for eco-efficient devices.

The implemented communication stack is also shown in Figure 3. In addition to the physical and link levels provided by the LPWAN transceivers and their corresponding firmwares, IPv6 connectivity has been integrated into the stack to enable the OBU's interoperability with Future Internet services and devices. For this to be done, an adaptation layer based on the Static Context Header Compression (SCHC) scheme has been developed as described in [38]. In the upper layer, applications based on Representational State Transfer (REST) over TCP/IP are supported, but the Constrained Application Protocol (CoAP) for restricted IoT communications is also considered.

With this architecture, the running software is in charge of aggregating data from sensors, deciding reporting interval, and choosing the most appropriate communication technology. For the case of LoRaWAN packets, they reach a LoRaWAN gateway that forwards the messages to a LoRaWAN server. The latter is in charge of extracting the data payload and sending it to the corresponding cloud service by using standard IoT protocols such as Message Queuing Telemetry Transport (MQTT). In turn, communications through the NB-IoT link are supported by the coverage provided by a cellular service provider and involves a series of 3GPP protocols that are transparent for the OBU, as they are already implemented in the NB-IoT transceiver. While LoRaWAN deployments are usually composed 
of private gateways and servers, NB-IoT ones depend on rigid telcos infrastructures and the traffic flows should cross their premises.

\subsection{Prototype}

The implemented OBU prototype is illustrated in Figure 4. As described above, it is composed of two principal components that are shown in Figure 4a. The main board is a Raspberry Pi Zero W, with a $1 \mathrm{GHz}$ ARM CPU and $512 \mathrm{MB}$ of RAM. In turn, the communication concentrator has been designed from scratch to integrate the desired transceivers. This board mounts an FTDI FT4232H chip to interface four serial ports from the USB interface provided by the Pi Zero. Therefore, the GPS receiver (Lantronix A2235-H), the LoRaWAN transceiver (Murata CMWX1ZZABZ-093), and the NB-IoT modem (Quectel BC95) are integrated into this board by using RS-232 ports. Typical $868 \mathrm{MHz}$ and multiband LTE stick antennas are attached to LoRaWAN and NB-IoT transceivers, respectively. An OLED display (SparkFun LCD-14532) is connected as well for outputting debugging and status messages, as can be seen in Figure $4 b$.

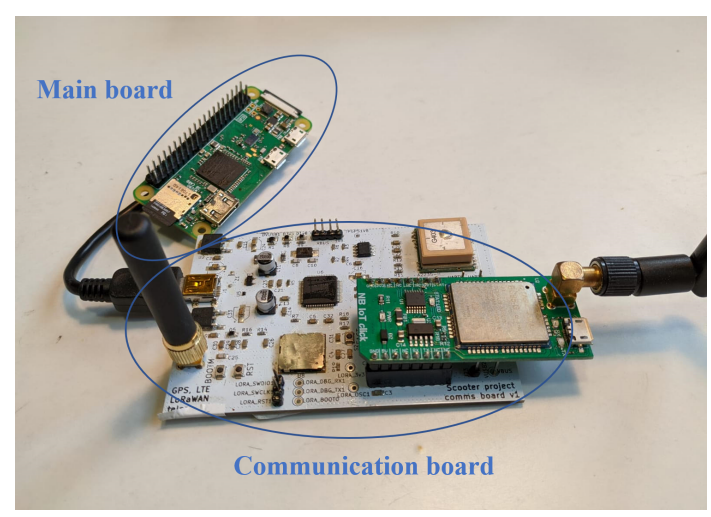

(a)

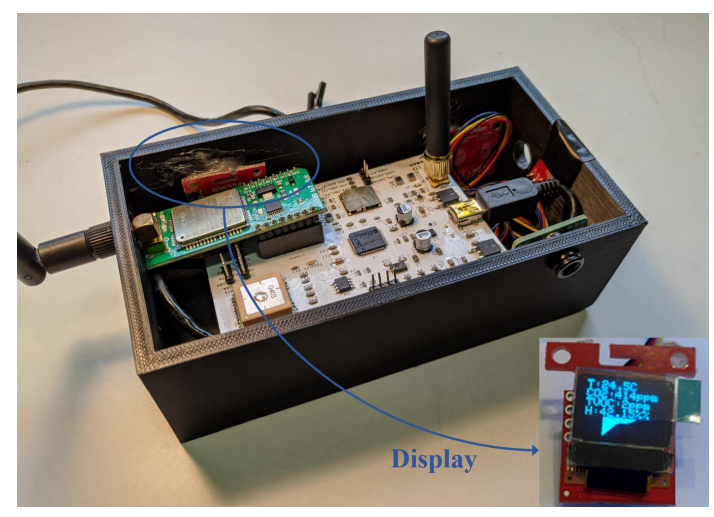

(b)

Figure 4. Implemented OBU prototype. (a) Main board and communication concentrator, (b) Unit installed in its case.

The prototype also includes a range of sensors attached to the Raspberry Pi Zero to enable smart city and C-ITS tracking and monitoring applications: an infra-red thermal array sensor $\left(\mathrm{CO}_{2}\right)$, an environment (pressure, humidity, temperature and $\mathrm{CO}_{2}$ ) unit (CCS811 and BME280) and a 9 DOF inertial sensor (LSM9DS1).

As shown in Figure $4 \mathrm{~b}$, the communication concentrator is placed over the main board and the sensors are distributed around the principal elements. Finally, a lithium power bank (RS PRO PB-A5200) with 5 Ah of capacity has been attached to the unit and placed below the boards. In our experiments, we have obtained a battery life time of more than two days, working at $5 \mathrm{~V}$ and reporting data regularly. The dimensions of the whole set are $15 \times 7 \times 5 \mathrm{~cm}$, and an adequate enclosure has been designed and 3D-printed for its easy integration on-board a bike or moped, or using a personal mobility device.

\subsection{Validation Tests}

In order to study the communication performance of our prototype, we have placed it aboard an electric scooter and travelled around the Espinardo Campus at the University of Murcia (Spain). These vehicles are a clear example of personal mobility device that can greatly improve urban mobility, but lack an external energy source and proper C-ITS integration. Together with e-bikes, e-scooters are flooding the urban landscape these days. In addition, the selected area for conducting our experiments presents realistic and heterogeneous conditions due to the presence of communication obstacles such as buildings, trees, etc. 
The data were retrieved from the OBU's embedded sensors and transmitted to a Graphana cloud server by using a developed Python script. For the data transmission, one or both of the available LPWAN links established with (i) a private LoRaWAN gateway (Kerlink Wirnet Station) installed in the campus or (ii) a cellular NB-IoT infrastructure deployed in the area by a telecommunications company (Vodafone) were employed.

The received data were stored in a database and showed in real time to the user by means of the dashboard presented in Figure 5. As observed, different temporal series are provided; hence, the user may access this information by using her smart device or a regular computer to check the current status or a historical record of the device parameters. In the specific example depicted in Figure 5, different plots representing network metrics such as throughput or packet loss can be seen, in this case extracted from the NB-IoT link. This information is relevant to evaluate the performance of the communications and detect disconnections or low coverage areas. Data extracted from some of the equipped sensors and transmitted over the LoRaWAN connection are also shown, namely, a plot representing the scooter's speed and another one showing the evolution of the detected level of $\mathrm{CO}_{2}$ during a ride. In this case, the unit was suddenly exposed to the exhaust pipe of a combustion motorbike to check the good operation of the sensor. In addition, other parameters such as GPS position, inertial sensor measurements, etc. are also transmitted to the cloud. All these raw data are stored in the server, so they are available to be employed by final services involving data processing or analytics. In this line, several applications like those identified in Section 3 may be developed. For example, we are currently working on an online vehicle tracking service to avoid thefts and the use of Machine Learning (ML) techniques for the detection of falling events by analyzing accelerometer data [39].

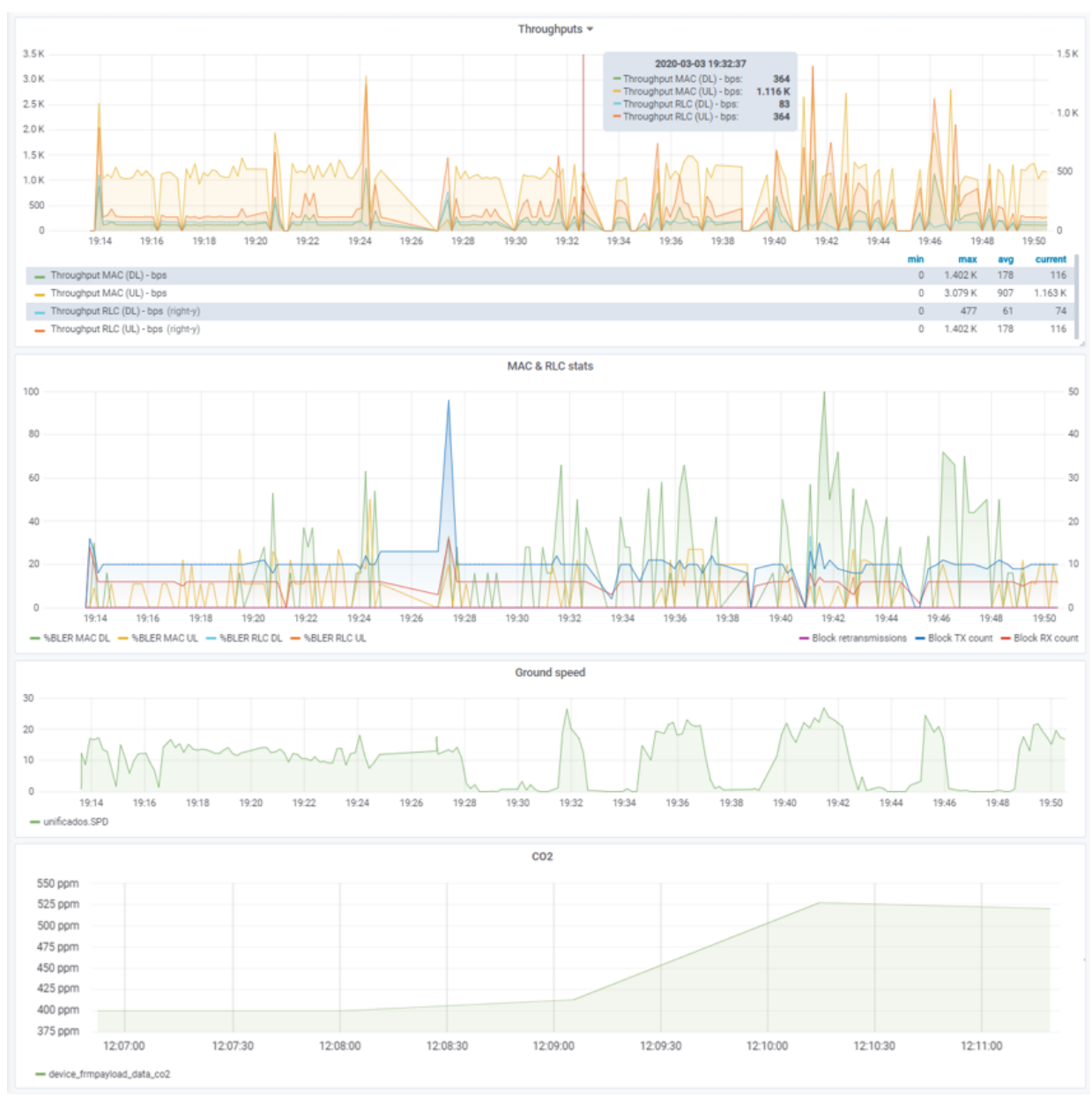

Figure 5. Data dashboard with results from validation tests. 


\subsection{Evaluation Tests}

Once we validated the functionality of our OBU prototype, we conducted a series of experimental tests in which we evaluated the performance of the two LPWAN technologies integrated into the unit. Concretely, we made some rides around the university campus ring to measure the Signal-to-Noise Ratio (SNR) obtained for both communication alternatives as well as additional network performance metrics such as Packet Delivery Ratio (PDR) and Round Trip Time (RTT).

Figure 6 presents two heat-maps indicating the real-time position of the device and the quality of the connection in each point in terms of Signal-to-Noise Ratio (SNR) for the LoRaWAN and NB-IoT links. Regarding the LoRaWAN experiment, a decay in the link quality in the southwest area of the campus can be noticed, due to some buildings that blocked the signal from the base station. This is proven by low SNR values and some noticeable packet losses (long gaps between consecutive successful transmissions). As mentioned above, our unit just connected to the unique LoRaWAN gateway installed by us in our facilities. On the other hand, in the case of NB-IoT, we could not control the base-station that the device was connected to, as this is an internal and automatic process managed by the communication interface. At the time of performing these tests, our NB-IoT service provider (Vodafone) had some base stations active surrounding the university campus. Even so, some lacks of coverage are also evidenced in the north and south areas. We expect this situation to improve by deploying additional gateways/base stations in the surrounding areas.

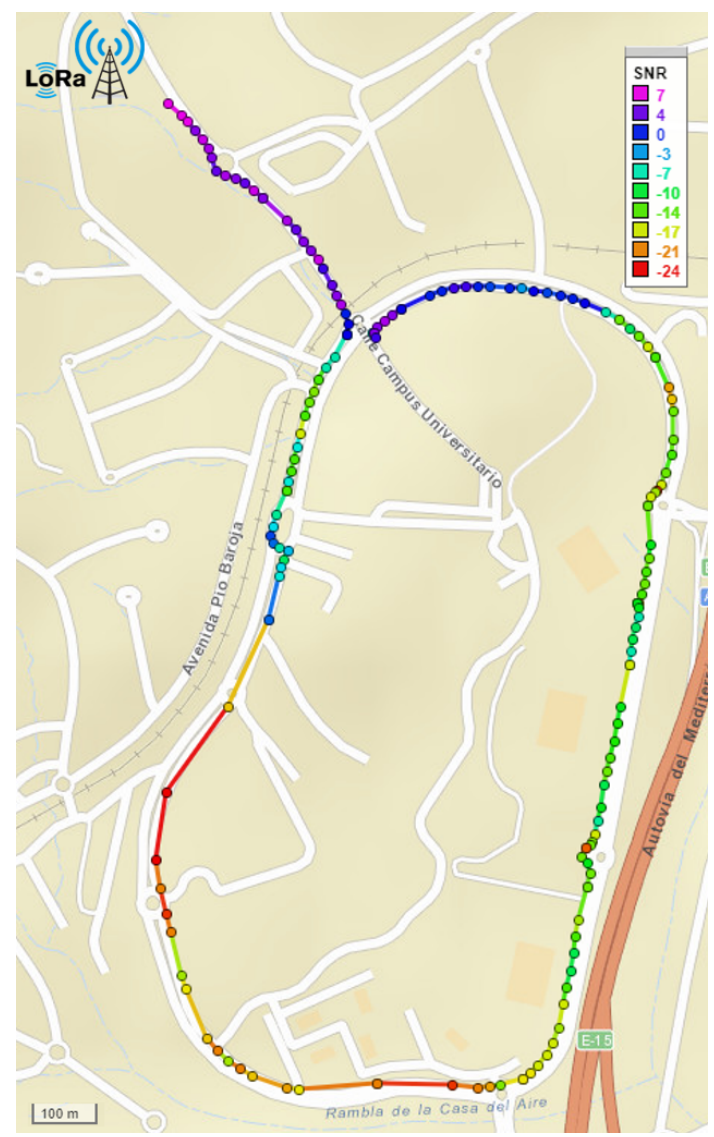

(a) LoRaWAN

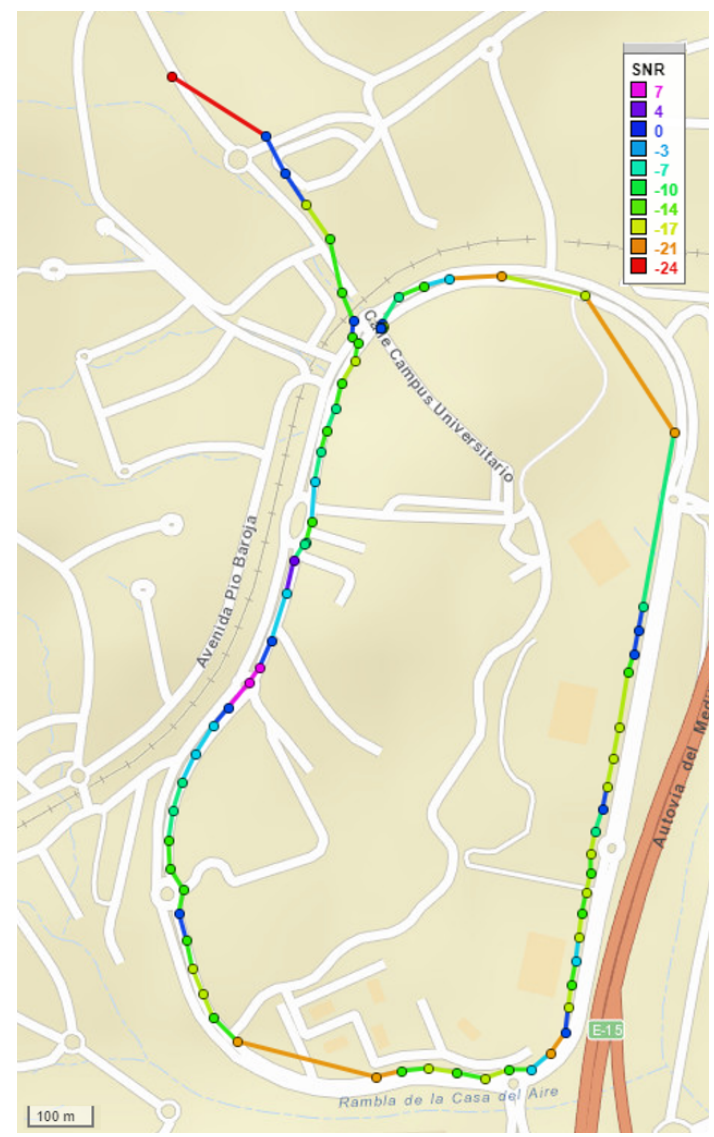

(b) NB-IoT

Figure 6. SNR obtained in one test ride around the campus ring.

From a network performance perspective, Table 1 shows a comparison between both LPWAN communication technologies, attending to SNR, PDR, RTT, and power consumption measurements. It can be observed that, although the average SNR attained for LoRaWAN is better than that 
obtained for NB-IoT, the evaluated network metrics demonstrate a superior performance of the latter. Focusing on the PDR, the packet loss recovery mechanisms implemented by NB-IoT permits to retransmit lost packets, allowing it to reach almost $100 \%$ of PDR. On the other hand, LoRaWAN does not implement any similar loss recovery scheme; hence, lower PDR values are obtained, although it is close to a notable figure of $80 \%$. The greatest difference between both technologies is evidenced in the measured RTTs. The shorter RTT achieved by NB-IoT is justified by its faster transmission data rate on the order of kilo bits per second (kbps), which is not heavily impacted by a longer network path through the operator' network. In turn, the conservative configuration selected in our experiments for LoRaWAN (Spreading Factor (SF) = 12) permits to establish more robust links at the expense of dramatically reducing the transmission rate down to just a few bits per second (bps). For this reason, the time-on-air taken by the transmitted packets (data payload of 50 bytes) is more than two seconds in each trip. Therefore, it can be concluded that, from a networking perspective, NB-IoT offers superior performance to LoRaWAN for this type of mobile setting.

Observing the energy consumption of both interfaces, it can be seen that the instant current requirements of NB-IoT are higher than the ones of LoRaWAN (data extracted from the specification sheet of each modem). This fact is even more exacerbated given the operational mechanisms of each technology. While NB-IoT requires a regular synchronization of the end-device with the infrastructure by means of the exchange of multiple control messages, LoRaWAN does not need such mechanism, so the number of transmissions and receptions are notably lower in this case. Therefore, it is evidenced that the superior communication capabilities provided by NB-IoT are achieved at the expense of greater power consumption. This is a key factor to be considered when designing OBUs devoted to battery-powered devices intended to operate during long time periods without external power supply. From our trials, consisting in rides of about $1 \mathrm{~h}$, we have measured a power consumption of approximately $300 \mathrm{mAh}$ for the whole prototype using both communication interfaces. Therefore, a typical powerbank of 10,000 mAh would imply an OBU working time over $33 \mathrm{~h}$, which gives room for a more intensive use of the unit. In addition, powerbanks of this capacity just add an extra payload of about $250 \mathrm{~g}$, which is a negligible figure for current personal mobility vehicles. The low power consumption of the $\mathrm{OBU}$, together with the enabled communication and processing capabilities, pave the way for the development of a new range of services involving eco-efficient vehicles integrated within future smart city environments.

Table 1. Network performance results.

\begin{tabular}{ccc}
\hline Metric & LoRaWAN & NB-IoT \\
\hline SNR (dB) & $-8.86 \pm 1.37$ & $-10.45 \pm 0.15$ \\
\hline PDR (\%) & $78.01 \pm 3.11$ & $99.3 \pm 0.38$ \\
\hline RTT (ms) & $460.21 \pm 5.31$ & $294.17 \pm 14.49$ \\
\hline \multirow{3}{*}{ Consumption } & TX: $106 \mathrm{~mA}$ & TX: $250 \mathrm{~mA}$ \\
& RX: $22 \mathrm{~mA}$ & RX: $50 \mathrm{~mA}$ \\
& Sleep: $1.65 \mu \mathrm{A}$ & Sleep: $2 \mathrm{~mA}$ \\
\hline
\end{tabular}

\section{Conclusions}

Urban mobility is experiencing a great change nowadays. City inhabitants are becoming more conscious about their carbon footprint so they are adopting more sustainable mobility options. Personal vehicles such as electric scooters, shared bikes, segways, etc. are massively populating our streets. However, these elements do not yet form part of the C-ITS and smart city ecosystems. Thus, in this paper, we have discussed the potential benefits and challenges to be tackled regarding the integration of these elements in future hyper-connected spaces. In addition, a novel OBU prototype for providing personal vehicles with connectivity has been presented. The attained results prove the validity of the proposal for the desired digitalization of this new wave of eco-efficient personal vehicles, 
given the communication capabilities provided by the developed OBU, which also presents a notably reduced power consumption. Communication performances are evaluated attending to the LPWAN technology used, obtaining interesting results that indicate that the communication solution should be carefully selected attending to the characteristics of the application to be implemented.

Within our own research lines, it is planned to widen the scope of the work by evaluating network performances using additional communication technologies, e.g., 5G, integrating big data analytics, and developing new services such as fall detection or advanced vehicle diagnosis, to improve urban mobility and safety as well as environmental preservation. In general, connectivity of personal mobility vehicles and mobile urban sensing open a research niche to be further explored in the upcoming years-from communication technologies to network protocols and from the integration of electronics considering power constraints to services exploiting data gathered. What is clear is the potential benefit expected from the proliferation of these connected electric vehicles for reducing carbon emissions and improving citizens' quality of life.

Author Contributions: Conceptualization, R.S.-I. and J.S.; Data curation, R.S.-I. and L.B.-E.; Funding acquisition, J.S.; Investigation, R.S.-I., L.B.-E., and J.S.; Methodology, R.S.-I. and J.S.; Project administration, R.S.-I. and J.S.; Validation, L.B.-E. and J.S.; Writing—original draft, R.S.-I., L.B.-E., and J.S.; Writing—review and editing, R.S.-I., L.B.-E., and J.S. All authors have read and agreed to the published version of the manuscript.

Funding: This work has been supported by the Spanish Ministry of Science, Innovation and Universities, under the Ramon y Cajal Program (Grant No. RYC-2017-23823) and the projects PERSEIDES (Grant No. TIN2017-86885-R with ERDF funds) and Go2Edge (RED2018-102585-T); by the European Commission, under the 5G-MOBIX (Grant No. 825496), IoTCrawler (Grant No. 779852) and the INSPIRE-5Gplus (Grant No. 871808) projects; and by the BBVA Foundation, under the 2018 Leonardo Grant for Researchers and Cultural Creators.

Conflicts of Interest: The authors declare no conflict of interest.

\section{References}

1. Teter, J.; Tattini, J.; Petropoulos, A. Tracking Transport 2020; Technical Report; International Energy Agency: Paris, France, 2020.

2. Dulebenets, M.A.; Moses, R.; Ozguven, E.E.; Vanli, A. Minimizing Carbon Dioxide Emissions Due to Container Handling at Marine Container Terminals via Hybrid Evolutionary Algorithms. IEEE Access 2017, 5, 8131-8147. [CrossRef]

3. Abioye, O.F.; Dulebenets, M.A.; Pasha, J.; Kavoosi, M. A Vessel Schedule Recovery Problem at the Liner Shipping Route with Emission Control Areas. Energies 2019, 12, 2380. [CrossRef]

4. Lo, P.L.; Martini, G.; Porta, F.; Scotti, D. The determinants of $\mathrm{CO}_{2}$ emissions of air transport passenger traffic: An analysis of Lombardy (Italy). Transp. Policy 2020, 91, 108-119. [CrossRef]

5. Gorner, M.; Teter, J. Electric Vehicles; Technical Report; International Energy Agency: Paris, France, 2020.

6. Wang, J.; Liu, J.; Kato, N. Networking and Communications in Autonomous Driving: A Survey. IEEE Commun. Surv. Tutor. 2019, 21, 1243-1274. [CrossRef]

7. Javed, M.A.; Hamida, E.B. On the Interrelation of Security, QoS, and Safety in Cooperative ITS. IEEE Trans. Intell. Transp. Syst. 2017, 18, 1943-1957. [CrossRef]

8. Sanchez-Iborra, R.; Cano, M.D. State of the art in LP-WAN solutions for industrial IoT services. Sensors 2016, 16, 708. [CrossRef] [PubMed]

9. Wang, J.; Jiang, C.; Zhang, K.; Quek, T.Q.S.; Ren, Y.; Hanzo, L. Vehicular Sensing Networks in a Smart City: Principles, Technologies and Applications. IEEE Wirel. Commun. 2018, 25, 122-132. [CrossRef]

10. Lee, U.; Gerla, M. A survey of urban vehicular sensing platforms. Comput. Netw. 2010, 54, 527-544. [CrossRef]

11. Wang, Y.; Chen, G. Efficient Data Gathering and Estimation for Metropolitan Air Quality Monitoring by Using Vehicular Sensor Networks. IEEE Trans. Veh. Technol. 2017, 66, 7234-7248. [CrossRef]

12. Briante, O.; Campolo, C.; Iera, A.; Molinaro, A.; Paratore, S.Y.; Ruggeri, G. Supporting augmented floating car data through smartphone-based crowd-sensing. Veh. Commun. 2014, 1, 181-196. [CrossRef]

13. Silva, M.; Signoretti, G.; Oliveira, J.; Silva, I.; Costa, D.G. A Crowdsensing Platform for Monitoring of Vehicular Emissions: A Smart City Perspective. Future Internet 2019, 11, 1. [CrossRef] 
14. Zuo, C.; Liang, K.; Jiang, Z.L.; Shao, J.; Fang, J. Cost-Effective Privacy-Preserving Vehicular Urban Sensing System. Pers. Ubiquitous Comput. 2017, 21, 893-901. [CrossRef]

15. Urra, O.; Ilarri, S. Spatial crowdsourcing with mobile agents in vehicular networks. Veh. Commun. 2019, 17, 10-34. [CrossRef]

16. Santos, P.M.; Rodrigues, J.G.P.; Cruz, S.B.; Lourenço, T.; d'Orey, P.M.; Luis, Y.; Rocha, C.; Sousa, S.; Crisóstomo, S.; Queirós, C.; et al. PortoLivingLab: An IoT-Based Sensing Platform for Smart Cities. IEEE Internet Things J. 2018, 5, 523-532. [CrossRef]

17. Masini, B.M.; Bazzi, A.; Zanella, A. Vehicular Visible Light Networks for Urban Mobile Crowd Sensing. Sensors 2018, 18, 1177. [CrossRef] [PubMed]

18. Cespedes, S.; Salamanca, J.; Yanez, A.; Vinasco, D. Group Cycling Meets Technology: A Cooperative Cycling Cyber-Physical System. IEEE Trans. Intell. Transp. Syst. 2019, 20, 3178-3188. [CrossRef]

19. Anaya, J.J.; Talavera, E.; Gimenez, D.; Gomez, N.; Felipe, J.; Naranjo, J.E. Vulnerable Road Users Detection Using V2X Communications. In Proceedings of the 2015 IEEE 18th International Conference on Intelligent Transportation Systems, Las Palmas, Spain, 15-18 September 2015; pp. 107-112.

20. Miucic, R.; Rajab, S.; Bai, S.; Sayer, J.; Funkhouser, D. Improving Motorcycle Safety through DSRC Motorcycle-to-Vehicle Communication; SAE Technical Paper; SAE International: Warrendale, PA, USA, 2015; pp. 1-8.

21. Chiu, P.J.; Tsai, H.-M. An intersection collision avoidance system for scooters utilizing non-line-of-sight links. In Proceedings of the 2012 IEEE Global Communications Conference (GLOBECOM), Anaheim, CA, USA, 3-7 December 2012; pp. 49-55.

22. Steltenpohl, H.; Bouwer, A. Vibrobelt: Tactile Navigation Support for Cyclists. In Proceedings of the 2013 International Conference on Intelligent User Interfaces; Association for Computing Machinery: New York, NY, USA, 2013; pp. 417-426.

23. Hernandez-Jayo, U.; De-la Iglesia, I.; Perez, J. V-Alert: Description and Validation of a Vulnerable Road User Alert System in the Framework of a Smart City. Sensors 2015, 15, 18480-18505. [CrossRef]

24. Vagnoli, C.; Martelli, F.; Filippis, T.D.; Lonardo, S.D.; Gioli, B.; Gualtieri, G.; Matese, A.; Rocchi, L.; Toscano, P.; Zaldei, A. The sensorwebbike for air quality monitoring in a smart city. In Proceedings of the IET Conference on Future Intelligent Cities, London, UK, 4-5 December 2014; pp. 1-4.

25. Aguiari, D.; Delnevo, G.; Monti, L.; Ghini, V.; Mirri, S.; Salomoni, P.; Pau, G.; Im, M.; Tse, R.; Ekpanyapong, M.; et al. Canarin II: Designing a smart e-bike eco-system. In Proceedings of the 2018 15th IEEE Annual Consumer Communications Networking Conference (CCNC), Las Vegas, NV, USA, 12-15 January 2018; pp. 1-6.

26. Almeida, R.; Oliveira, R.; Luís, M.; Senna, C.; Sargento, S. A Multi-Technology Communication Platform for Urban Mobile Sensing. Sensors 2018, 18, 1184. [CrossRef]

27. Department of Economic and Social Affairs. World Urbanization Prospects; Technical Report; United Nations: New York, NY, USA, 2018.

28. Santa, J.; Fernández, P.J. Seamless IPv6 connectivity for two-wheelers. Pervasive Mob. Comput. 2017, 42, 526-541. [CrossRef]

29. Esztergár-Kiss, D.; Csiszár, C. Evaluation of Multimodal Journey Planners and Definition of Service Levels. Int. J. Intell. Transp. Syst. Res. 2015, 13, 154-165. [CrossRef]

30. Nuzzolo, A.; Comi, A. Real-time modelling of normative travel strategies on unreliable dynamic transit networks: A framework analysis. In Modelling Intelligent Multi-Modal Transit Systems; Nuzzolo, A., Lam, W.H.K., Eds.; CRC Press, Taylor \& Francis Group: Boca Raton, FL, USA, 2017; Chapter 5, pp. 130-151.

31. Nuzzolo, A.; Persia, L.; Comi, A.; Polimeni, A. Shared Autonomous Electrical Vehicles and Urban Mobility: A Vision for Rome in 2035. In Data Analytics: Paving the Way to Sustainable Urban Mobility; Nathanail, E.G.; Karakikes, I.D., Eds.; Springer International Publishing: Cham, Switzerland, 2019; pp. 772-779.

32. David, K.; Flach, A. CAR-2-X and Pedestrian Safety. IEEE Veh. Technol. Mag. 2010, 5, 70-76. [CrossRef]

33. Jin, W.L.; Kwan, C.; Sun, Z.; Yang, H.; Gan, Q. SPIVC: A smartphone-based inter-vehicle communication system. In Proceedings of the Transportation Research Board 91st Annual Meeting, Washington, DC, USA, 22-26 January 2012; pp. 1-12.

34. Malone, K.M.; Soekroella, A.M. Estimating benefits of C-ITS deployment, when legacy roadside systems are present. IET Intell. Transp. Syst. 2019, 13, 915-924. [CrossRef] 
35. Seo, H.; Lee, K.D.; Yasukawa, S.; Peng, Y.; Sartori, P. LTE evolution for vehicle-to-everything services. IEEE Commun. Mag. 2016, 54, 22-28. [CrossRef]

36. Campolo, C.; Molinaro, A.; Iera, A.; Fontes, R.R.; Rothenberg, C.E. Towards 5G Network Slicing for the V2X Ecosystem. In Proceedings of the 4th IEEE Conference on Network Softwarization and Workshops (NetSoft), Montreal, QC, Canada, 25-29 June 2018; pp. 400-405. [CrossRef]

37. Cailean, A.M.; Cagneau, B.; Chassagne, L.; Popa, V.; Dimian, M. A survey on the usage of DSRC and VLC in communication-based vehicle safety applications. In Proceedings of the IEEE 21st Symposium on Communications and Vehicular Technology in the Benelux (SCVT), Delft, The Netherlands, 10 November 2014; pp. 69-74. [CrossRef]

38. Sanchez-Gomez, J.; Gallego-Madrid, J.; Sanchez-Iborra, R.; Santa, J.; Skarmeta Gómez, A.F. Impact of SCHC Compression and Fragmentation in LPWAN: A Case Study with LoRaWAN. Sensors 2020, 20, 280. [CrossRef] [PubMed]

39. Ren, L.; Peng, Y. Research of Fall Detection and Fall Prevention Technologies: A Systematic Review. IEEE Access 2019, 7, 77702-77722. [CrossRef]

Publisher's Note: MDPI stays neutral with regard to jurisdictional claims in published maps and institutional affiliations.

(C) 2020 by the authors. Licensee MDPI, Basel, Switzerland. This article is an open access article distributed under the terms and conditions of the Creative Commons Attribution (CC BY) license (http:/ / creativecommons.org/licenses/by/4.0/). 